\title{
Erratum to: Regional cerebral blood flow in late-onset schizophrenia: a SPECT study using 99mTc-ECD
}

\author{
Rei Wake ${ }^{1}$ Tsuyoshi Miyaoka ${ }^{1}$ Tomoko Araki ${ }^{1}$ Kazunori Kawakami ${ }^{1}$. \\ Motohide Furuya $^{1} \cdot$ Erlyn Limoa $^{1} \cdot$ Sadayuki Hashioka $^{1} \cdot$ Jun Horiguchi $^{1}$
}

Published online: 16 June 2015

(C) Springer-Verlag Berlin Heidelberg 2015

\section{Erratum to: Eur Arch Psychiatry Clin Neurosci DOI 10.1007/s00406-015-0607-z}

The authors regret the errors that occurred in the official published Tables 4 and 5. Unfortunately, these tables are duplication of Table 6 in the online published article. The correct version of Tables 4 and 5 are given below.

Table 4 Areas of decreased rCBF in early-onset schizophrenia and control-1

\begin{tabular}{llrrrrl}
\hline$k$ value & $Z$ score & $x(\mathrm{~mm})$ & $y(\mathrm{~mm})$ & $z(\mathrm{~mm})$ & BA & Localization \\
\hline 2696 & 4.69 & 50 & -2 & 2 & 22 & rt. sup. temporal gyrus \\
1265 & 4.08 & -56 & -14 & 12 & 42 & lt. trans. temporal gyrus \\
598 & 4.05 & 8 & -20 & -10 & 25 & rt. orbital gyrus \\
223 & 3.65 & 0 & -10 & 10 & $*$ & lt. anterior cingulate \\
54 & 3.58 & -8 & 10 & lt. thalamus \\
\hline
\end{tabular}

The $k$ value, $Z$ score, and Talairach coordinates of peak voxels are provided for each cluster $(Z$ score $>3, P$ voxel level $<0.001 ; P$ cluster level $<0.05$, corrected for multiple comparisons)

*No gray matter found

Table 5 Areas of decreased rCBF in late-onset schizophrenia and control-2

\begin{tabular}{|c|c|c|c|c|c|c|}
\hline$k$ value & $Z$ score & $x(\mathrm{~mm})$ & $y(\mathrm{~mm})$ & $z(\mathrm{~mm})$ & BA & Localization \\
\hline 4881 & 6.01 & -40 & 14 & -8 & 13 & lt. insular \\
\hline 3615 & 6.00 & 44 & 14 & -2 & 47 & rt. inf. frontal gyrus \\
\hline 654 & 4.54 & -30 & 24 & 52 & 8 & lt. sup.frontal gyrus \\
\hline 2057 & 4.49 & 0 & 46 & 16 & 9 & lt. med. frontal gyrus \\
\hline 475 & 4.04 & -2 & 12 & -8 & 25 & 1t. anterior cingulate \\
\hline 136 & 3.99 & -40 & -44 & -34 & $*$ & lt. cerebellar tonsil \\
\hline 361 & 3.91 & 20 & 16 & 66 & 6 & rt. sup. frontal gyrus \\
\hline 280 & 3.83 & 0 & -18 & 12 & 2 & lt. thalamus \\
\hline
\end{tabular}

The $k$ value, $Z$ score, and Talairach coordinates of peak voxels are provided for each cluster $(Z$ score $>3, P$ voxel level $<0.001 ; P$ cluster level $<0.05$, corrected for multiple comparisons)

*No gray matter found

The online version of the original article can be found under doi:10.1007/s00406-015-0607-z.

Rei Wake

rei@med.shimane-u.ac.jp

1 Department of Psychiatry, Shimane University Faculty

of Medicine, Izumo, Japan 